\title{
Impact of glycoscience in fighting Covid-19
}

\author{
Roberto Adamo ${ }^{1}$. Sandro Sonnino ${ }^{2}$
}

Published online: 5 June 2020

(C) Springer Science+Business Media, LLC, part of Springer Nature 2020

It is a long time that the glycobiology rightfully entered the world of biomedical sciences. Nevertheless, it remains a science for a few insiders, remaining largely associated only with food, nutrition and diets for the majority of people and many scientists.

One of the main goal of Glycoconjugate Journal and of the Authors that publish their results in it, is to present to the scientific world that carbohydrates are a key point for the very existence of the cellular system. Proteins are often glycoproteins, and many lipids are glycolipids. The glycans linked to proteins and lipids are multiple structures and play fundamental roles in the incredibly vast world of "good and bad" cellular processes governed by fundamental weak interactions.

In many cases these processes are "good" for bacteria and viruses that thank to carbohydrate-carbohydrate interactions infect our body, but are "bad" for us. This is well associated with the ongoing Covid-19 (COronaVIrus Disease 19) pandemic that is strongly impacting on our society, with more than $5.4 \mathrm{M}$ cases and $343 \mathrm{~K}$ deaths recorded world-wide at the time this editorial is written. Numbers that could grow by an order of magnitude before the virus can be eradicated.

Covid-19 has triggered with impressive speed the research of an ample arsenal of different therapeutic approaches to combat its causative agent SARS-Cov-2 (Severe Acute Respiratory Syndrome Coronavirus 2).

It is plausible that different strategies will contribute to eventually defeat the Covid-19: small anti-viral molecules are being tested to block the infection, and the nucleotide analogue Remdesivir has recently been approved by FDA as "standard of care" for patients with COVID-19 [1]. Other

Roberto Adamo

roberto.x.adamo@gsk.com

Sandro Sonnino

sandro.sonnino@unimi.it

GSK, Siena, Italy

2 Department of Medical Biotechnology and Translational Medicine, University of Milan, Milan, Italy therapeutic approaches, such as purified plasma and monoclonal antibodies will help the immune system to counteract the virus.

A vaccine will be key to prevent the infection and allow society to go back to "normality," yet we really need to learn some lessons out of the pandemic about preparedness in limiting their spread in the future. There are currently 8 vaccine candidates in clinical trial and more than 100 potential vaccines at preclinical stage [2].

Along with classic inactive viral vaccines, modern approaches based on subunit proteins, nucleic acids and viral vectors for the delivery of the target antigen are advancing in the clinical trial [3]. In all these efforts major focus has been directed to the trimeric spike glycoprotein $\mathrm{S}$, which mediates cell entry [4]. This protein is composed of the two subunit S1 and S2 that are not covalently associated before fusion to the target cells, and are generated from $\mathrm{S}$ through a proteolytic cleavage site. After the virion internalization by the host, conformational changes occurring as a consequence of the cleavage by endo-lysosomal proteases at level of the S2' cleavage site, allows for the fusion activation of the coronavirus $\mathrm{S}$ proteins and interactions with the host receptors [5]. To accomplish this function, SARS-CoV-2 spike protein binds to its receptor human ACE2 (hACE2, human angiotensin converting enzyme 2) through its receptor-binding domain (RBD) and is proteolytically activated by human proteases [6].

Structural and sequence differences among the regions predicted to be immunoreactive and bind/elicit antibodies render different the S spike glycoprotein from SARS-Cov and the more recent SARS-Cov-2 [7].

The $\mathrm{S}$ protein presents multiple variably glycosylated sites [8] and in silico 3D simulations have predicted them to form a dense coating on its surface [9], whose role on the protein conformation and immune evasion is currently under investigation and deserves to be fully understood.

Glycosylation of viral envelope proteins is known to be exploited by the pathogen to escape recognition by the host immune system by masking relevant protein epitopes from detection by antibodies, and can influence the ability of the 
host to raise an effective adaptive immune response or even enhance the viral infectivity.

It seems that the SARS-Cov-2 surface, in spite of its dense carbohydrate layer, could offer some areas of vulnerability, as observed for SARS and MERS [10]. On the other hand, a potent neutralizing antibody binds to a glycosylated epitope, indicating that sugars are not insignificant decorations of the protein surface [11].

Therefore, it remains a relevant question whether the glycosylation of the $\mathrm{S}$ protein in coronavirus plays a fundamental role in evasion as in HIV or it is less impactful allowing sufficient exposition of peptide epitopes as for the influenza virus. All this suggests to have great care on the glycans of spike, as this understanding could guide developing a vaccine capable to eradicate the SARS-Cov-2 and close the pandemic Covid-19.

Glycans are key players not only in the viral surface envelope but also on the binding receptor. Some of the coronavirus (specifically the so called $\beta 1$-Covs) employ glycan-based receptors carrying 9-O-acetylated sialic acid $\left(\mathrm{Neu} 5,9 \mathrm{Ac}_{2}\right)$ for binding of the $\mathrm{S}$ protein [12]. Binding to sialic acids has been shown to be involved in promotion of the infection, also supporting the intercellular expansion of $\mathrm{CoV}$ infections and could be involved in their transition from zoonotic to human infections [13].

Finally it should not be disregarded the role of glycans, as mucin components, in preserving a proper protective functionality of the lung protective mucosa, providing a physical barrier and clearing pathogens, but also mediated immunological and inflammatory responses in opposition to the viral infection [14].

\section{Compliance with ethical standards}

Conflict of interest RA is an employee of GSK group of companies.

\section{References}

1. Wang, Y., Zhang, D., du, G., du, R., Zhao, J., Jin, Y., Fu, S., Gao, L., Cheng, Z., Lu, Q., Hu, Y., Luo, G., Wang, K., Lu, Y., Li, H.,
Wang, S., Ruan, S., Yang, C., Mei, C., Wang, Y., Ding, D., Wu, F., Tang, X., Ye, X., Ye, Y., Liu, B., Yang, J., Yin, W., Wang, A., Fan, G., Zhou, F., Liu, Z., Gu, X., Xu, J., Shang, L., Zhang, Y., Cao, L., Guo, T., Wan, Y., Qin, H., Jiang, Y., Jaki, T., Hayden, F.G., Horby, P.W., Cao, B., Wang, C.: Remdesivir in adults with severe COVID19: a randomised, double-blind, placebo-controlled, multicentre trial. Lancet. 395, 1569-1578 (2020). https://doi.org/10.1016/S01406736(20)31022-9

2. https://www.who.int/who-documents-detail/draft-landscape-ofcovid-19-candidate-vaccines

3. Callaway, E.: Nature. 580, 577 (2020)

4. Walls, A.C., Tortorici, M.A., Bosch, B.J., Frenz, B., Rottier, P.J.M., DiMaio, F., Rey, F.A., Veesler, D.: Cryo-electron microscopy structure of a coronavirus spike glycoprotein trimer. Nature. 531(7592), 114-117 (2016)

5. Wrapp, D., Wang, N., Corbett, K.S., Goldsmith, J.A., Hsieh, C.L., Abiona, O., Graham, B.S., McLellan, J.S.: Cryo-EM structure of the 2019-nCoV spike in the prefusion conformation. Science. 367(6483), 1260-1263 (2020)

6. Shang, J., Wan, Y., Luo, C., Ye, G., Geng, Q., Auerbach, A., Li, F.: Cell entry mechanisms of SARS-CoV-2. Proc. Natl. Acad. Sci. U. S. A. 117, 11727-11734 (2020). https://doi.org/10.1073/pnas. 2003138117

7. D'Annessa, et al.: BioRxiv. (2020). https://doi.org/10.1101/2020. 03.13.990267

8. Shajahan, et al.: bioRxiv. (2020). https://doi.org/10.1101/2020.04. 01.020966

9. Grant, et al.: bioRxiv. (2020). https://doi.org/10.1101/2020.04.07. 030445

10. Watanabe, et al.: BioXriv. (2020). https://doi.org/10.1101/2020.02. 20.957472

11. Pinto, et al.: bioRxiv. (2020). https://doi.org/10.1101/2020.04.07. 023903

12. Hulswit, R. J. G., et al., (2019) 116 (7), 2681

13. Qing, E., et al.: mBio. 11(1), e02764 (2020)

14. Denneny, E., Sahota, J., Beatson, R., Thornton, D., Burchell, J., Porter, J.: Mucins and their receptors in chronic lung disease. Clin Transl Immunology. 9(3), e01120 (2020)

Note Some of the information here reported derives from BioRxiv that is a preprint server where reports are not peer-reviewed.

Publisher's note Springer Nature remains neutral with regard to jurisdictional claims in published maps and institutional affiliations. 